\title{
Contractus originarius: la justicia como gramática de la democracia
}

\section{Contractus originarius: justice as a grammar of democracy}

\section{MIGUEL FONSECA MARTÍNEZ}

Doctor en Filosofía Universidad Santo Tomás Institución Universitaria Colegios de Colombia (Unicoc), Colombia dirhumanidades@unicoc.edu.co ORCID ID: https://orcid.org/0000-0003-4398-7346

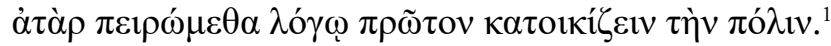




\section{Resumen}

La noción de justicia se refiere sinópticamente a las formas más convenientes del trato que se da a los individuos y a la manera en que este surge y se fundamenta en el trasfondo de la sociedad. El enfoque normativo de la justicia constituye el concepto de lo justo, en tanto exista una adecuación entre las normas de una teoría de la justicia específica y las conductas de los individuos respecto de esta. Este enfoque teórico abre la posibilidad de una fundamentación racional sobre las creencias y el razonamiento político. Un análisis epistémico cumpliría una tarea importante y posiblemente fundante de una crítica a lo que consideramos el trato justo entre individuos, colectivos y pueblos. Este artículo comprende la justicia como el conjunto de elementos normativos de carácter formal, atados a la regulación epistémica, que constituyen modelos del trato justo de individuos y colectividades. La justicia es así un nivel metanormativo de las instituciones políticas. A partir de un contrato originario, aquel que se constituye en la coherencia entre la normatividad epistémica o interna de los individuos, el modelo de justicia y su aplicación, se permitiría la construcción de una sociedad justa.

Palabras clave: justicia, democracia, política, contractualismo, normatividad epistémica.

\section{Abstract}

The notion of justice refers synoptically to the most convenient forms of treatment given to individuals and to the way in which it arises and is based on the background of society. The normative approach to justice represents the concept of fairness, as long as there is an adequacy between the norms of a specific theory of justice and the behavior of individuals with respect to it. This theoretical approach opens the possibility of a rationale on beliefs and political reasoning. An epistemic analysis would fulfill an important and possibly foundational task of a critique of what we consider to be fair treatment between individuals, groups and peoples. This article addresses justice as the set of normative elements of a formal nature, tied to epistemic regulation, which constitute models of the fair treatment of individuals and communities. Justice is thus a meta-normative level of political institutions. The building of a just society would be possible as far as we start from an original contract, one that is constituted in the coherence between the epistemic or internal normativity of the individuals, the model of justice and its application.

Keywords: justice, democracy, politics, contractualism, epistemic normativity. 


\section{Introducción}

La noción de justicia se refiere sinópticamente a las formas más convenientes del trato que se da a los individuos y, por ello, a la manera en que dicho trato surge y se fundamenta en el trasfondo de la sociedad. Esta noción ha sido aplicada como un criterio axiológico a las acciones individuales, a las políticas públicas e, incluso, a las leyes positivas en su sentido correctivo y a la relación de estas con la distribución de recursos y la constitución de estructuras institucionales. Entre los diferentes enfoques teóricos que han dado cuenta de su definición y aplicabilidad, existe un conjunto de posturas con cierto aire de familia que quisiera denominar teorías con un enfoque normativo de la justicia. Tal aire de familia en ciertas aproximaciones teóricas de estudio se caracteriza principalmente por su intención de formalizar los criterios o leyes epistémicas que configuran la normatividad a la cual se deben atener las diversas particularizaciones del trato justo a los individuos y colectivos humanos. Los hitos fundamentales de este enfoque, Kant y Rawls, serán analizados como las premisas fundamentales del argumento que se propone.

El enfoque normativo de la justicia constituye el concepto de lo justo, en tanto exista una adecuación entre las normas de una teoría de la justicia específica y las conductas de los individuos respecto de esta, en un marco social y, por tanto, político. En esta lectura, el concepto de virtud moral desempeña un papel tangente o subsidiario al citado compromiso con una estructura normativa epistémica.

Por consiguiente, se deriva la posibilidad de una fundamentación racional sobre las creencias y el razonamiento político. Tal enfoque normativo brindaría a la razón humana horizontes para constituir cierta normatividad conformada por estructuras modélicas. Ellas surgen a partir de la soberanía de los criterios epistémicos propios de la racionalidad inherente a los individuos y a las colectividades que les dan subsistencia. Un análisis epistémico cumpliría una tarea importante y posiblemente fundante de una crítica a lo que consideramos el trato justo entre individuos, colectivos y pueblos.

Se comprende la justicia desde este enfoque como el conjunto de elementos normativos de carácter formal, atados a la regulación epistémica, que constituyen modelos del trato justo de individuos y colectividades. La justicia es así un nivel metanormativo de las instituciones políticas. A partir de un contrato originario, aquel que se constituye en la coherencia entre la normatividad epistémica o interna de los individuos, el modelo de justicia y su aplicación, se permitiría la construcción de una sociedad justa. Por esto, en un primer momento, el contrato es un experimento del pensamiento, un contrafáctico, un escenario epistémico modal, en el cual se ponen a prueba las proposiciones metanormativas y normati- 
vas que pueden constituir la sociedad y los criterios de justicia, con la pretensión de grados altamente plausibles de generalidad. Los criterios que se infieren de este experimento epistémico conforman un horizonte de la acción política que, tendiendo a la universalidad, pretenden como entelequia del trato justo una convivencia pacífica de los pueblos.

Surge así, como propósito principal de reflexión, la necesidad de delinear los primeros trazos de un posible modelo o teoría de la normatividad interna o epistémica, que permita formular cierto tipo de estándares objetivos para la acción práctica y la toma de decisiones colectivas. A partir de este boceto, podríamos comprender el concepto de justica, usando la metáfora wittgensteineana, como una gramática de la democracia; un lecho dinámico del río que fundamenta y modela el trato justo de la humanidad.

Para constituir estos primeros trazos, se describirá en un primer momento el origen de este enfoque en el pensamiento, pionero, fundante y en algún caso insuperable de Kant, sobre el particular. Ulteriormente, se hablará de la formulación de esta perspectiva normativa en el siglo XX, principalmente, a partir de los postulados de Rawls y su revisión de las ideas kantianas. Finalmente, en un tercer momento, tras develar los principales argumentos del enfoque normativo, se mostrará la necesidad de un nuevo contrario originario que considere como fundamento un análisis de la normatividad epistémica que consolida el lecho del río de una teoría sobre la justicia. Se hará así patente la necesidad de construir una teoría sobre la normatividad interna o epistémica como agenda necesaria para construir una sólida teoría de la justicia que apela a un fuerte trabajo trasdisciplinar con la epistemología y la lógica.

\section{Origen de una teoría constructiva y normativa de la justicia en el pensamiento de Kant}

\section{Sobre la naturaleza del derecho en Kant}

El propósito fundamental de la filosofía política kantiana es realizar una defensa de la Ilustración a partir del análisis especializado de la idea de libertad. Desde su primera instrucción filosófica, Kant recibió el legado de los ideales de la Ilustración a través de la filosofía de Wolff: "Wolff's philosophy had a profound sym- 
bolic significance in early eighteenth century Germany: It represented the very vanguard of the Auflärung, the attempt to establish the authority of reason in all walks of life, wheter in the state, the church, the universities or society at large" (Beiser, 1992, p. 29).

Las primeras intuiciones filosóficas le llevaron a pensar que la única salvación para la Ilustración en contra del pietismo imperante era la construcción de una metafísica robusta que, a través de una justificación racional para nuestras creencias morales, permitiera un justo medio entre el escepticismo y el fideísmo pietista.

Sin embargo, la lectura de Rousseau lo lleva más prontamente a la idea de un fundamento de la filosofía práctica en la idea de libertad. Afirma en este sentido: "Ich würde mich unnützer finden wie den gemeinen Arbeiter wenn ich nicht glaubete dass die Betrachtung allen übringen einen Werth ertheilen könne, die rechte der Menschheit herzustellen"3 (Kant, 2008a: Ak 20: 44).

Tras este giro en su pensamiento, Kant comprenderá el fundamento de la moral y la filosofía práctica en la libertad en sí misma; la buena voluntad será la fuente de las leyes prácticas. La metafísica, en sentido tradicional, hacía patente el problema de buscar la fuente de la moralidad y la filosofía práctica en el mundo externo, y así renunciar a los poderes que nos permite el ejercicio de la libertad:

The previous history of practical philosophy foundations and first principles were sought in objective ideas, in a normative constitution of the cosmos, in the will of God, in the nature of man, or in prudence in the service of self interest; but Kant was convinced that these starting-points were without exeption inadequate for the foundation of unconditional practical laws, and the human reason could only concede absolute practical neccesity and obligatorieness to norms that arose from its own legislation. (Kersting, 1992, p. 342)

Los seres humanos estamos sujetos, según estas ideas, solo a las leyes de la razón. Así, el derecho brota como un principio necesariamente objetivo, eterno, válido y universal, con acceso irrestricto al conocimiento de cualquier ser humano. Gracias a

2 La filosofía de Wolff tuvo una profunda significatividad simbólica en la filosofía alemana del siglo dieciocho: Esta representa la más pura vanguardia de la llustración, el intento de establecer la autoridad de la razón en todos los aspectos de la vida, entre ellos, en el Estado, la Iglesia, las universidades y la sociedad en general.

3 Me sentiría más inútil que el trabajador común si no creyera que estas consideraciones pueden aportar algún mérito a los demás, es decir, establecer los derechos del hombre. 


\section{0 | Miguel Fonseca Martínez \\ Contractus originarius: la justicia como gramática de la democracia}

él, se pueden hacer patentes los criterios de corrección y juzgamiento de las acciones humanas. Deviene de ello que el concepto de derecho no puede constituirse desde ningún punto de vista empírico ni atado a intencionalidades particulares basadas en apetitos. Kant define el concepto de derecho de la siguiente forma:

Das Recht ist also der Inbegriff der Bedingungen, unter denen die Willkür des andern nach einen allgemeinen Gesetze der Freiheit zusammen vereinigt werder kann. Eine jede Handlung ist Recht, die oder nach deren Maxime die Freiheit der Willkür eines jedem mit jedermanns Freiheit nach einem allgemeinen Gesetze zusammen bestehen kann. ${ }^{4}$ (Kant, 2008b: 6: 230)

Solo los efectos de las acciones sobre la libertad de acción de los otros le incumben al derecho; las intenciones inherentes a los particulares y sus convicciones, intereses y necesidades son excluidas, por tanto, de la esfera de la justicia. El derecho no tendría como finalidad ayudar a los débiles y sus necesidades. El derecho consistiría más bien en la posibilidad de protección entre aquellos que tienen la libre voluntad de actuar.

La ley del derecho desde la razón es una ley formal universal de la libertad de acción; se relaciona solo con la compatibilidad formal de la libertad externa de una persona para con las otras. El derecho permite identificar la posibilidad de una distribución inequitativa de la libertad.

Tal derecho en su sentido externo permite la distribución de la libertad a seres ubicados, incluso, espacialmente y, por tanto, plantea los límites y la defensa de aquello que conviene al ejercicio de esta libertad. Por esa razón, el concepto de derecho para Kant puede ser planteado como un principio de coerción: "Princip der Möglichkeit eines äusseren Zwanges, der mit der Freiheit von jedermannn nach allgemeinen Gezetzen zusammen bestehen kann"5 (Kant, 2008b: Ak 6:232).

El concepto universal de derecho se constituye en una analogía simétrica del imperativo categórico; permite establecer unos límites entre las acciones de todos, a través de cierto tipo de modelos de correspondencia y compatibilidad entre la

4 El derecho es también el modelo de condición bajo el cual la voluntad de una persona puede ser unificada con la voluntad de otra bajo la ley universal de libertad. Toda acción es derecho, la cual permite que la libertad de la voluntad de cada cual permanezca junto a la libertad de todos.

5 La posibilidad de comprender la coerción externa bajo el asentimiento de la libertad de cada cual, de acuerdo con leyes universales. 
porción de libertad asignada a cada individuo de una forma a priori por su condición misma de ser humano.

En la relación que el derecho establece con las citadas condiciones externas de la existencia humana, surge el concepto de propiedad. El derecho a la propiedad deviene la posibilidad de tener dominio y autoridad sobre los objetos y, según el principio negativo de coerción, abre la posibilidad de excluir a los demás del uso o soberanía sobre estos objetos. El conjunto de cosas no son objeto de derecho, pero sí son susceptibles de ser usadas y poseídas por el hombre según conveniencia. El uso de las cosas solo puede ser delimitado por la ley formal del derecho en un ejercicio de la razón:

Kant's foundation of private property therefore implies the autorization for original acquisition. To be sure, it is at first difficult to see how such an original acquisition can possibly be rightful: Empirical acts of appropiation cannot constitute any right, and unilateral acts of will cannot generate any sort of obligation. If all obligations arose either naturally or through contract or promise, then there would be no way for original acquisition to give right to any obligation. Kant's solution of this difficulty consists in the apparently paradoxical construction of a noncontractualistic theory of consensus. ${ }^{6}$ (Kersting, 1992, p. 350)

La teoría de Kant comparte así cierta similitud con la teoría de Locke (2003) respecto de la primera adquisición de la propiedad a través del trabajo, en tanto se establece previamente a cualquier forma de contrato, pero la rechaza dado que las acciones empíricas, como se decía, no generan derecho, al igual que, de paso, el trabajo no produce ningún tipo de obligación. La exclusión que causa el concepto de propiedad privada necesita una autorización consensuada. Tal consenso deviene las condiciones a priori de la razón respecto del derecho, el imperativo categórico del derecho, que nos hace pensar en un sujeto ideal universal que debería poseer empíricamente los objetos en virtud de su libertad. No obstante, de ello se desprende el principio negativo coercitivo ya señalado, según el cual se

6 "La Fundamentación kantiana de la propiedad privada, por lo tanto, implica la autorización para la adquisición original. Sin duda, es difícil de ver inicialmente como tal adquisición original puede ser correcta: Los actos empíricos de apropiación no pueden constituir ningún derecho, y los actos unilaterales de la voluntad no pueden generar ninguna suerte de obligación. $\mathrm{Si}$ todas las obligaciones surgen o bien naturalmente o a través de un contrato o promesa, entonces no habría forma para una adquisición original que dé derecho a alguna obligación. La solución de Kant a esta dificultad consiste en la aparentemente paradójica construcción de una no contractualista teoría del consenso". (Kersting, 1992, p.350) 


\section{2 | Miguel Fonseca Martínez \\ Contractus originarius: la justicia como gramática de la democracia}

hace indispensable la confirmación judicial positiva, es decir, el paso de lo que se ha denominado el estado de naturaleza al Estado o mundo civil (Rauscher, 2016).

\section{El contrato como principio práctico necesario}

Kant comprende el estado de naturaleza como una condición del derecho natural privado. Afirma en ese sentido:

Aus dem Privatrecht im natürlichen Zustande geht nun das Postulat des öffentlichen Rechts hervor: du sollst im Verhältnisse eines unvermeidlichen Nebeneinandersseins mi tallen anderen aus jenem heraus in einen rechtlichen Zustaand, d.i. den einer austheilenden Gerechtigkeit übergehen. ${ }^{7}$ (Kant, 2008b: Ak 6: 307)

El derecho debe ser positivo, concreto e institucionalizado, enmarcado en una justicia distributiva. El estado de naturaleza es de esta suerte un conjunto caótico privado de interpretaciones sobre la manera en que la libertad de poseer puede ser entendida. El postulado del derecho público emana de la razón, para evitar las múltiples representaciones del asunto.

Debido a esto, la idea del Estado surge igualmente como una condición necesaria de la razón práctica. Gracias a criterios universales, el Estado armoniza las diversas representaciones de la libertad, a la manera de un acuerdo. Así, los individuos ya están delimitados por la razón misma para abandonar el estado de naturaleza:

Der Act wodurch sich das Volk selbst zu einem Staat constituirt, eigentlich aber nur die Idee desselben, nach der die Rechtmässigkeit desselben allein gedacht werder kann, ist der ursprüngliche Contract, nach welchem alle im Volk ihre äusere Freiheit aufgaben, um sie als Glieder eines gemeinen Wesens, d.i. desVolks als Staat betrachtet. ${ }^{8}$ (Kant, 2008b: Ak 6: 315)

7 Desde el derecho privado en la condición natural surge el postulado del derecho público: en relación con la ineludible coexistencia con otros, se hace una transición de estado de naturaleza al estado jurídico, es decir, uno de justicia distributiva.

8 El acto a través del cual el pueblo se constituye a sí mismo en un Estado, o más bien solo la idea de tal acto, de acuerdo con el cual la legalidad del Estado puede ser concebida, es el contrato original según el cual todos en el pueblo rinden su libertad externa en orden a recuperarla como miembros de una comunidad, sea la del pueblo considerado Estado. 
La necesidad de un contrato originario es consecuente con una idea de la historia construida desde la mera razón; a partir del concepto racional de derecho, los seres humanos se constituyen en sociedad. El contrato racional es, por tanto, una condición obligatoria para cualquier acaecimiento histórico, es decir, más allá de cualquier determinación empírica como formas violentas de dominación, tiranías, etc.

La aplicación del contrato no requiere ningún sesgo voluntarista, sino simplemente el experimento propio del pensamiento que nos permite la universalización, en imitación evidente al proceso que, según Kant, se lleva a cabo en el ámbito de la razón moral. Este experimento del pensamiento es muy parecido a lo que se puede denominar un escenario epistémico modal, en el cual se prueban la posibilidad y necesidad de las proposiciones relativas al concepto de derecho.

Der bürgerliche Zustand also, bloss als rechlicher Zustand betrachtet, ist auf folgende Principien a priori gegründet:

1. Die Freiheit jedes Gliedes der Societät, als Menschen.

2. Die Gleichheit desselben mit jedem Anderen, als Unterhan.

3. Die Selbstständigkeit jedes Gliedes eines gemeinen Wesens, als Bürgers. ${ }^{9}$ (Kant, 2008, c: Ak 8:290)

La consecuencia evidente de estos supuestos es la sujeción en las prácticas regladas solo a escenarios necesarios y universales. Sin embargo, no se puede decir que Kant construye, como se ve, una metafísica del derecho; la filosofía del derecho en Kant se compromete, en cierto sentido, con la historia y su reforma (Langer, 1986). Kant comprende que la realización del derecho, la libertad y la razón en general, podríamos decir también la realización de la Ilustración, tienen lugar en un mundo concreto atado a condiciones históricas. En el tiempo de Kant, el escenario de la República podría ser la mejor simulación de un estado ideal de democracia o Estado de derecho en sentido estricto.

La entelequia de la simulación de la República sería un orden internacional de derecho, en que sean el caso las condiciones antedichas, más allá de la idea de

9 La condición civil, considerada como condición de derecho, está basada a priori en los siguientes principios: a) la libertad de todos sus miembros en tanto humanos, b) la igualdad de cada miembro con todo otro y c) la autosuficiencia de cada miembro de la comunidad como ciudadano. 


\section{4 | Miguel Fonseca Martínez \\ Contractus originarius: la justicia como gramática de la democracia}

Estados nacionales y todas las ataduras empíricas que conllevan (Höffe, 2002). El objetivo del derecho es la paz mundial más allá de la idea del Estado nación.

\section{La paz perpetua como idea irrealizable}

La paz perpetua se constituye en una idea necesaria para guiar la acción política:

Nun hat aber die republikanische Verfassung ausser der Lauterkeit ihres Ursprungs, aus dem reinen Quell des Rechtsbegriffs entsprungen zu sein, noch die Aussicht in die gewünche Folge nämlich den ewingen Frieden; wovon der Grund dieser ist. Wenn die Beistimmung der Staatsbürger dazu erfordert wird, um zu beschliessen, ob Krieg über sich selbst beschliessen müsten. ${ }^{10}$ (Kant, 2008c: Ak 8: 351)

La paz es una posibilidad, un contrafáctico, el mundo posible producto de la razón ilustrada que gobierna las acciones humanas. Este argumento puede ser clarificado a través del famoso ejemplo de Platón en el Laques: un soldado entenderá como poco razonable que su participación en un ejercicio militar a gran escala sea realmente relevante; empero, es más probable que si permanece en la milicia pueda ser herido o dado de baja. Por tanto, la toma de decisión racional del soldado será desistir de ir a la guerra y escapar (Platón, 2007). Si tal escenario de la batalla Delia narrada por Sócrates se universaliza, entonces no hay lugar para la guerra y el ejercicio de derecho racional implicaría una desobediencia civil que ni siguiera permite la revolución en el entorno de los Estados nacionales (Rawls, 1971).

Como se puede ver, toda la arquitectura de la filosofía del derecho de Kant surge y termina en una metafísica del derecho, es decir, en un conjunto de escenarios epistémicos modales. Entre ellos, la idea de la paz perpetua es, quizá, la mejor muestra. Kant acepta de entrada su carácter irrealizable: "So ist der ewige Friede (das letze Ziel des ganzen völkerrechts) freilich eine unausführbare Idee"11 (Kant, 2008b: Ak 6: 350).

El ejercicio y las tareas epistémicas de la razón pueden ser inferidos en general de este análisis de la metafísica del derecho kantiana; nos afirma en la concepción

10 Ahora, para clarificar más el origen puro del concepto de derecho, la constitución republicana tiene, además, la perspectiva de alcanzar su resultado deseado, a saber, la paz perpetua; el fundamento es el siguiente: se requiere el consentimiento del ciudadano para decidir el decreto de guerra sobre sí mismo.

11 Así, la paz perpetua (que es la finalidad del derecho de gentes) es una idea inviable. 
del pensamiento como un ejercicio de reflexión lógica y consistente de alto nivel, que construye mundos posibles como guías de la acción práctica.

La paz perpetua como un ejercicio de metafísica modal se constituye en una típica formulación de entidades subsistentes que permiten horizontes al pensamiento para la toma de decisiones en espacios concretos, y en referencia a la manera en que nos relacionamos con los hechos en bruto.

\section{Enfoque constructivo y normativo de la justicia: Kant revisited}

El enfoque normativo comprende la justicia como el conjunto de elementos normativos de carácter puramente formal, que, además de regular las instituciones políticas, les confiere estructura y poder. Es, por tanto, como se ha hecho evidente, heredero del constructivismo moral y la deontología kantiana (Rawls, 1980). El origen de las normas es el consenso (Mejía, 2008). Este no tiene como fundamento ningún tipo de principio moral o religioso, es decir, metafísico, sino que brota de un horizonte vital, histórico y de cierta cultura política que determina el acaecimiento, la necesidad y el carácter del acuerdo, si bien se desprende de estas contingencias en el momento en que tal consenso se sedimenta en el lenguaje (Mejía, 2011, p. 144). En tanto asunto esencialmente lingüístico, el contrato que constituye tales estructuras normativas no es definitivo; el consenso está atado a la discusión pública y, por tanto, se relaciona con otras formas de normatividad, a saber, la regulación epistémica, lógica, argumentativa, lingüística y retórica del lenguaje (Feldman, 1991).

Puede apuntalarse mejor esta definición afirmando que la justicia, bajo el enfoque normativo, es un nivel metanormativo de las instituciones políticas. La justicia es una estructura regulativa que determina la forma en que se instauran derechos y deberes; beneficios y responsabilidades (Cárdenas y Guarín, 2010, pp. 213-217). Bajo esta perspectiva, las libertades fundamentales son un mínimo que no puede subsumirse a la conveniencia o utilidad; los principios liberales básicos son un supuesto que aparece en el trasfondo del acuerdo y garantiza la posibilidad de la realización de un plan particular y racional de vida. Los principios liberales permiten igualmente intencionalidades y comportamientos cooperativos para la obtención de bienes sociales primarios (Rawls, 2013, pp. 270-339). 


\section{6 | Miguel Fonseca Martínez \\ Contractus originarius: la justicia como gramática de la democracia}

El consenso requiere una situación inicial que supone condiciones normales de comportamiento humano cooperativo; si se quiere, la idea normalizada de ciudadano. Los ciudadanos deben aparecer en iguales condiciones para eliminar desigualdades y descender al máximo la suerte que cada quien padece.

La justicia es una justicia equitativa que, en el acuerdo básico de la vida con otros, busca mejorar las condiciones de comportamiento cooperativo como elemento necesario para constituir particularidades vitales. Se sigue que de los procedimientos, de tipo universal, surjan resultados materiales de tipo particular. Las desigualdades materiales surgen por causas atadas al trasfondo del acuerdo, es decir, a la cultura política y económica de los pueblos. Por ello, el enfoque normativo de la justicia privilegia y defiende la institucionalidad, al igual que el imperio de la ley.

El enfoque normativo no solo comprende la justicia en el ámbito del Estado nación, sino también en el del derecho de gentes (Pogge, 2002; Rawls, 2001). Realizando una proyección de los fundamentos teóricos enunciados, se podría pensar en una posible justicia global basada en un constitucionalismo democrático de los pueblos.

La idea de justicia en el enfoque normativo, dado que está anclada a la idea de constructivismo politico, requiere igualmente un fuerte concepto de razón pública (Rawls, 2013, pp. 204-238). Por ello, un análisis de la normatividad epistémica podría dar cuenta de todas aquellas bisagras que constituyen el trasfondo del acuerdo y su estructura esencial (Siegel y Biro, 1997).

El objetivo fundamental de este constructivismo político, y su exigencia de normatividad, es establecer estándares objetivos para la acción práctica y la toma de decisiones colectivas. Estos criterios de objetividad surgen de la inconmensurabilidad de los diferentes contextos culturales, morales y sociales. El pluralismo inherente a los diferentes mundos de la vida exige una revisión de los criterios, fundamentos y normatividad para la toma de decisiones colectivas.

De esto se sigue que uno de los problemas fundamentales del constructivismo político contemporáneo, heredero de la filosofía práctica kantiana, es su concomitante normatividad racional, lógica y discursiva. Por ello, deviene como una posible clave para el desarrollo y la crítica de esta postura, un análisis de la normatividad epistémica que soporta como pilar fundamental la idea de justicia. Revisar la normatividad epistémica de la acción colectiva puede modelar más precisamente la justicia como principio metanormativo de la razón práctica. 


\section{El lecho del río: justicia y normatividad epistémica}

\section{Normatividad epistémica de la acción colectiva}

A partir de lo dicho, surge la posibilidad de pensar en una normatividad interna o epistémica que permita establecer estándares objetivos para la acción práctica y la toma de decisiones colectivas; un contrato originario que emana de una deliberación interna que se atiene a dicha normatividad epistémica.

En este sentido, al teorizar sobre la naturaleza de elementos sociales como la acción colectiva, los movimientos sociales, las revoluciones sociales y nociones como libertad y justicia, se apela a normas, reglas y valores como posibles elementos con carácter constitutivo y explicativo. La normatividad no puede ser ignorada, ya que estos fenómenos y nociones, en tanto productos de la mente humana y relativos al ámbito de lo humano, dependen de la forma en que los individuos los constituyen como realidad (Risjord, 2014, p. 8). Los compromisos ontológicos sobre entidades como la justicia, por lo menos en un primer momento, recaen, por tanto, sobre la mente humana. Estos seres subsisten a través del lenguaje por las elaboraciones de los procesos mentales humanos y se rigen por las normas que cobijan los mecanismos epistémicos desde donde surge su subsistencia.

Así, el centro de la cuestión gira en torno a la normatividad. Esta se refiere en general a dos ámbitos, a saber: el de nuestras acciones, que se identifica con la pregunta ¿qué debo hacer?, y el de nuestras creencias, que se identifica con la pregunta ¿qué debo creer? Ello deviene una normatividad teórica y una normatividad práctica. La primera asume, principalmente, el problema de la normatividad respecto de la posibilidad de hacer buenas o malas inferencias. La segunda, por su parte, discurre en torno a la maximización de la utilidad, la teoría de juegos, la teoría de la decisión racional y los criterios éticos y morales (Spohn, 2013).

Siguiendo a Hart (1997), existiría a su vez una perspectiva externa e interna sobre esta doble faz de la normatividad. Desde la primera, se establecen las normas que un sujeto adopta en una comunidad. Una norma en este sentido puede comprenderse como cierto tipo de regularidad convencional de comportamiento que soporta estructuras modélicas de intercambios de creencias y deseos en comunidades determinadas (Bloor, 1997; Lewis, 1969). La perspectiva externa entien- 


\section{8 | Miguel Fonseca Martínez \\ Contractus originarius: la justicia como gramática de la democracia}

de estas normas como hechos empíricos relativos a investigaciones empíricas que permiten encontrar las normas que regulan una comunidad.

La normatividad interna es, por otro lado, de otra índole, pues a los seres humanos les es inherente la consideración normativa sobre sus creencias y acciones; a esto se refiere tener una perspectiva interna sobre la normatividad. Al pensar si hacemos justicia o no, estamos guiados por razones, y apelamos a la normatividad de las razones, que, si bien no siempre son subsumidas a una necesidad lógica, están atadas a elementos plausibles de validez y, por ende, a reglas sobre el conocimiento y la razón (Spohn, 2013).

A esto que Hart (1997) denomina normatividad interna se le puede reconocer como normatividad epistémica de la justicia, es decir, el conjunto de reglas que me permiten configurar previamente cualquier normatividad externa, una deliberación interna que establezca los criterios y la validez de mis razones. El contrato originario consistiría en establecer a través de una deliberación interna, es decir, la escogencia de los mejores criterios de racionalidad, los fundamentos de cualquier consenso o disenso ulterior.

Lo anterior requiere la tarea de la formulación de una teoría modélica de la normatividad interna que no solo se encargue de afirmar a través de una recolección de datos aquello que una comunidad cree que es correcto o bueno; una teoría interna de la normatividad debe decir qué es ser correcto, algo que solo pude ser investigado, como se dice, desde una perspectiva interna relativa al ser humano y su estructura de racionalidad. Así, los cambios y estructura de la sociedad, y de cualquier otro producto de la mente humana, podrían asirse más fácilmente si se da cuenta de qué es correcto o incorrecto, en relación con la concepción de conceptos fundamentales que guían como un horizonte la acción colectiva.

Tal teoría modélica permitiría establecer los criterios de una deliberación interna que constituya el contrato originario a través del cual se permita una ulterior deliberación democrática que posibilite la construcción de la realidad social e institucional. Dicha teoría modélica permitiría fijar la forma de los argumentos que ulteriormente, al llenarse de contenido, configurarían los bloques que conforman la democracia. Esta relación entre justicia y epistemología daría bases más fuertes a este criterio regulador. 


\section{Normatividad interna y constructivismo}

Las relaciones entre este proyecto de formular una teoría de la normatividad interna, y la idea de constructivismo que se ha venido describiendo, encuentran un vínculo fuerte en el denominado constructivismo humeano:

In contrast to kantians, humean constructivists offer a more relativistic account of the nature of normative truths, according to which the truth of a normative claim consists in its being entailed from the evaluative stand point of particular individuals. The starting point of rational deliberation are contingent commitments, and practical concerns of actual agents. ${ }^{12}$ (Bagnoli, 2017)

La verdad de las proposiciones constitutivas de las estructuras sociales son relativas a agendas y agentes que evalúan diferentes alternativas racionales que compiten para dar solución a asuntos prácticos. La universalidad de la normatividad se convierte en una normatividad contingente que, sin embargo, no excluye la posibilidad de encontrar generalizaciones, es decir, iteraciones e invariabilidades respecto de la normatividad interna.

Los usos de las proposiciones normativas no pueden desligarse de los contextos en los que tienen lugar. Sin embargo, fuera del conjunto de proposiciones con sentido y uso práctico cotidiano, existen una cantidad de bisagras que configuran el uso mismo de estas herramientas epistémicas y lingüísticas (Stroll, 1994). A esto lo podemos denominar la gramática o normatividad interna que condiciona cualquier tipo de deliberación ulterior sobre la constitución de la realidad social.

Las proposiciones gramaticales, es decir, el conjunto de proposiciones sobre la normatividad interna, describirían prácticas que permiten esclarecer nuestra formación de conceptos. El ejemplo de Wittgenstein es esclarecedor:

Una pregunta filosófica es similar a una pregunta sobre la constitución de una determinada sociedad. Y sería como si una sociedad se constituyese sin reglas escritas claras, pero con una necesidad de ellas; de hecho con un instinto de acuerdo con el cual observasen (respetasen) ciertas reglas en sus reuniones; solo que esto resulta

12 En contraste con los kantianos, los constructivistas humanos ofrecen una propuesta un poco más relativista sobre la naturaleza de las verdades normativas, de acuerdo a la cual la verdad de una pretensión normativa consiste en ser inferida desde el punto de vista evaluativo de individuos particulares. El punto de vista de una deliberación racional son compromisos contingentes, y preocupaciones de agentes reales. 
difícil porque no hay nada claramente expresado sobre ello y no se ha establecido ninguna disposición que clarifique (ponga claramente de relieve) las reglas (Wittgenstein, 2015, p. 177)

Una de las tareas principales de la filosofía consistiría en mostrar ese conjunto de reglas que fijan la forma en que se constituyen los sistemas de creencias, las sociedades y las instituciones. El lenguaje como institución fundamental para la creación de la sociedad es un conjunto de juegos de lenguaje con gramáticas o lógicas regionales que obedecen a los requerimientos de diversos mundos de la vida colectiva. La intencionalidad colectiva y la acción colectiva fijan sus creencias en estos escenarios epistémicos normativos que configuran a la sociedad, según el mundo de la vida y la gramática del lenguaje al cual pertenece el colectivo que la está construyendo, a partir de acuerdos, consensos y contratos.

El comportamiento de este fundamento es, no obstante, dinámico. Siguiendo a Wittgenstein (2015), podemos decir que este conjunto de bisagras se comportan como el lecho de un río. Si bien todas las dinámicas del río van más rápido que el lecho, y si bien el lecho es fundamento, también tiene cierta forma de movimiento. La gramática de los juegos de lenguaje es y no es algo fijo. Por esta razón, se puede tener una visión dinámica de la justicia.

La tarea, pues, consistirá en formular una teoría modélica de esta normatividad interna o epistémica, necesariamente atada al concepto de racionalidad derrotable. El razonamiento monotónico, propio de los sistemas clásicos del razonamiento deductivo, del cual, por ejemplo, han bebido las teorías de Kant y la filosofía del derecho de Rawls, es aquel en el cual, si se tiene un argumento válido sin importar cuántas premisas nuevas puedan añadírsele, tal argumento mantiene su estatus de validez. El razonamiento práctico, en tanto forma de razonamiento que se encamina a optar por un curso prudente de acción en virtud de un objetivo, y usado en el contexto del diálogo y la deliberación, es dinámico y, por tanto, tiende a ser no monotónico. Las inferencias no monotónicas o formas derrotables de inferencia son tipos argumentativos en que se trazan conclusiones tentativamente, con la posibilidad de retractarse de ellas dada nueva información (Walton, 1996). La normatividad epistémica, y el proyecto de una normatividad sobre esta, debería atarse así a tal concepto de racionalidad no monotónica o derrotable, para permitir un lecho del río estable y al mismo tiempo dinámico. 


\section{Justicia como gramática de la democracia}

Usando la metáfora wittgensteineana, la justicia puede ser definida como la gramática de la democracia. El conjunto de prácticas de uso de proposiciones descriptivas sobre la acción colectiva, al igual que el conjunto de proposiciones que objetivan los hechos institucionales (proposiciones atadas a nuestros diversas formas de la vida particulares), fluyen y cambian como el agua del río sobre el trasfondo relativamente fijo del lecho del mismo río, que podemos parangonar con la normatividad interna que constituye la formación de la sociedad y produce el concepto de justicia. La justicia es algo así como el lecho del río de la democracia.

La relación entre los conceptos de justicia y democracia es sistemática y orgánica. La teoría democrática contemporánea define su carácter esencial como cierto tipo de método de decisión definido por el reconocimiento de la igualdad de los participantes en función de una toma de decisión colectiva (Christiano, 2006). La democracia extiende la idea de un dominio agencial, propio de la vida individual, al dominio extendido de las decisiones colectivas, es decir, una variación que permite un dominio extendido sobre el ambiente próximo.

Se piensa que existe una forma de consenso razonable en el cual, dando justificación a las creencias personales, existe coherencia y consistencia con una posible toma de decisión colectiva, más allá de que ella logre, por decirlo de algún modo, ser actualizada o institucionalizada. La democracia es equitativa en tanto se trata al otro como un agente razonable, y no porque produzca un consenso ideal. La democracia se relaciona con formas de consenso traslapado cuando los agentes sociales buscan tomar decisiones razonables sobre qué es lo mejor por hacer para su vida colectiva.

La justicia, según lo dicho, es un instrumento que busca garantizar una buena estructura en la toma de decisiones democráticas, pero no puede ser en sí misma entendida como un límite al ejercicio de la democracia (Hart, 1997). La justicia es más bien un producto del diseño político que equilibra los valores democráticos (Dworkin, 2010). Podemos decir que la justicia explicita la democracia, y la representatividad democrática se hace patente a su vez en los procesos institucionales de la justicia (Hart, 1997, pp. 128-133). Los ejercicios procesales facilitan y encauzan la toma de decisiones democráticas, no ejercen en sí mismos coerción sobre la acción política, sino que deben convertirse en facilitadores de su ejercicio.

La justicia puede ejercer con pertinencia tales tareas de fundamento dinámico si se entiende como un conjunto de proposiciones gramaticales o bisagras que 


\section{2 | Miguel Fonseca Martínez \\ Contractus originarius: la justicia como gramática de la democracia}

modelan y delimitan la creación de las instituciones y los marcos epistémicos agenciales de toma de decisiones democráticas. Estos goznes devienen los diversos mundos de la vida colectiva que requieren la construcción de instituciones y la toma de decisiones democráticas. Por tanto, una de las tareas prioritarias de una filosofía política o teoría política consistirá en establecer cuáles son estos goznes, cuándo les conviene ser aceitados y finalmente reemplazados según de las situaciones agenciales de los colectivos.

\section{Conclusiones}

La justicia se define como el conjunto de elementos de carácter formal, atados a la regulación epistémica, que constituyen modelos del trato adecuado que deberían recibir individuos y colectividades. En este sentido, dichos modelos pueden ser comprendidos como contrafácticos que surgen a partir de las diversas gramáticas de la racionalidad humana. Por tanto, la regulación epistémica de la racionalidad que aceptan los colectivos se configura como el verdadero contrato originario que da vida a una institucionalidad justa. El análisis de la estructura epistémica de los modelos de justicia permitiría a su vez entender cómo subsisten y funcionan. De esto deviene la necesidad de plantear teorías que den cuenta de la normatividad interna de nuestra racionalidad y permitan acuñar ulteriormente modelos de justicia pertinentes. Una teoría de la justicia fuerte requiere así trabajar mancomunadamente con la argumentación, la lógica y la epistemología, para lograr una revisión de los criterios que fundan una noción tan importante para la filosofía práctica. Este es el horizonte trazado para el diseńo de mejores modelos del trato adecuado que merece la humanidad.

\section{Referencias}

Aristóteles. (1998). Metafísica. Gredos.

Bagnoli, C. (2017, 23 de junio). Constructivism in metaethics. The Stanford Encyclopedia of Philosophy. https://plato.stanford.edu/archives/fall2017/entries/constructivismmetaethics/

Beiser, F. (1992). Kant's intellectual development (1746-1781). Cambridge University Press.

Bernal Pulido, C. (2010). Problemas de la posición de América Latina en el contexto de la globalización. En M. Carbonell y L. García Jaramillo (eds.), El canon neoconstitucional (pp. 592-612). Universidad Externado de Colombia Editores. 
Bloor, D. (1997). Wittgenstein, rules and institutions. Routledge.

Cárdenas Sierra, C. A. y Guarín Ramírez, E. A. (2010). Filosofía política y del derecho: Tomás de Aquino en diálogo con Bobbio, Chomsky, Rawls y Alexy. Universidad Santo Tomás.

Christiano, T. (2006, 27 de junio). Democracy. The Stanford Encyclopedia of Philosophy. https://plato.stanford.edu/archives/spr2015/entries/democracy/

Coliva, A. (2015). Extended rationality: A hinge epistemology. MacMillan.

Collingwood, R. (1947). The new leviathan. Clarendon.

Durkheim, E. (1964). Rules of sociological method. Free Press.

Dworkin, R. (2010). Igualdad, democracia y constitución: Nosotros, el pueblo, en los tribunales. En M. Carbonell Sánchez y L. García Jaramillo (eds.), El canon neoconstitucional (pp. 117-152). Universidad Externado de Colombia.

Feldman, S. (1991). The new metaphysics: The interpretative turn in jurisprudence. lowa Law Review, 76, 661-700.

Fraser, N. (2008). Escalas de justicia. Herder.

Frege, G. (1984). Collected papers on mathematics, logic and philosophy (B. F. McGuinness, ed.). Blackwell.

Gómez Alonso, M. M. (2006). Frágiles certidumbres: Wittgenstein y sobre la certeza, duda y lenguaje. Universidad Pontificia Salamanca.

González, A. (2015a). Para una ontología de lo social. Revista Portuguesa de Filosofía, $71,4,231-247$.

González, A. (2015b). El surgir de la ética. Ápeiron: Estudios de Filosofía, 3, 833-54.

González, A. (2015c). Surgimiento. Universidad Santo Tomás.

Haroche, C. (2015). Lo inevaluable en una sociedad de desconfianza. Universidad de Bogotá Jorge Tadeo Lozano.

Hart, J. (1997). Democracia y desconfianza. Siglo del Hombre.

Höffe, O. (2002). Kant's cosmopolitan theory of law and peace. Cambridge University Press.

Kant, I. (2008a). Beobachtungen über das Gefühl des Schönen und Erhabenen. Akademieausgabe von Immanuel Kants Gesammelten Werken.

Kant, I. (2008b). Die Metaphysic der Sitten. Akademieausgabe von Immanuel Kants Gesammelten Werken.

Kant, I. (2008c). Zum ewingen Frieden. Akademieausgabe von Immanuel Kants Gesammelten Werken.

Kersting, W. (1992). Politics, freedom, and order. Cambridge University Press.

Langer, C. (1986). Reform nach Prinzipien. Klett-Cotta Verlag.

Lewis, D. (1969). Convention: A philosophical study. Harvard University Press.

Locke, J. (2003). Two treatises of gobernment. Cambridge University Press.

Meinong, A. (1921). Über Gegenstand theorie: Selbstdarstellung. F. Meiner.

Mejía, A. (2011). Rawls, de la justicia al derecho como sujeto político. Ideas y Valores, 60(147), 143-152. http://www.scielo.org.co/scielo.php?script=sci_arttext\&pid =S0120-00622011000300008

Mejía Quintana, O. (2008). El paradigma consensual del derecho en la teoría de la justicia de John Rawls. En J. Rawls, El derecho de los pueblos. Tercer Mundo.

Platón. (2000). Leyes. En Diálogos. Gredos.

Platón. (2007). Laques. Gredos. 
Pogge, T. (2002). Cosmopolitanism: A defence. Critical Review of International Social and Political Philosophy, 5(3), 86-91. https://doi.org/10.1080/13698230410001702672

Rauscher, F. (2016, 1 de septiembre). Kant social and political philosophy. The Stanford Encyclopedia of Philosophy. https://plato.stanford.edu/entries/kant-social-political/

Rawls, J. (1971). A theory of justice. Harvard University Press.

Rawls, J. (1980). Kantian constructivism in moral theory. The Journal of Philosophy, 77(9), 515-572. https://doi.org/10.2307/2025790

Rawls, J. (2001). El derecho de los pueblos. Paidós.

Rawls, J. (2013). Liberalismo político. Fondo de Cultura Económica.

Risjord, M. (2014). Philosophy of social science. Routledge.

Searle, J. (1995). The construction of social reality. Free Press

Searle, J. (2010). Making the social world: The structure of human civilization. Oxford University Press.

Sellars, W. (1968). Science and metaphysics. Reidel.

Seumas, M. (2019, 9 de abril). Social institutions. The Stanford Encyclopedia of Philosophy. https://plato.stanford.edu/entries/social-institutions/

Siegel, H. y Biro, J. (1997). Epistemic normativity, argumentation, and fallacies. Argumentation, 17(3), 277-292. https://doi.org/10.1023/A:1007799325361

Sosa, E. (2011). Knowing full well. Princeton University Press.

Spohn, W. (2013). The laws of belief: Ranking theory and its philosophical applications. Oxford University Press.

Stroll, A. (1994). Moore and Wittgenstein on certainty. Oxford University Press.

Tomasello, M. (2009). The origins of human communication. MIT Press.

Tuomela, R. (2007). The philosophy of sociality: The shared point of view. Oxford University Press.

Walton, D. (1996). Argumentation schemes for presumptive reasoning. Lawrence Erlbaum Associates.

Williamson, T. (2013). Modal logic as metaphysics. Oxford University Press.

Wittgenstein, L. (2015). Observaciones filosóficas. En Filosofía: Secciones 86-93 del Big Typescript "secciones 86-93 del Big Typescript" (pp. 171-189). Tecnos.

Zalta, E. (1996). Principia Metaphysica. Stanford University. 